lates. Mutations in $r r l$ and $r r s$ genes were not detected. AST for 4 MAM isolates confirmed that inducible resistance is not present even with erm(41) T28 mutation. AST for 3 MAA with erm(41) C28 polymorphism showed MIC values below $2 \mathrm{mg} / \mathrm{L}$ which is interpreted by CLSI guidelines as sensitive strain. AST showed that MIC values for amikacin are between $8-16 \mathrm{mg} / \mathrm{L}$ interpreted as sensitive and concordant with molecular analysis.

In Slovenia, for macrolide and aminoglycoside resistance, phenotypic and genotypic results of Mycobacterium abscessus complex are concordant. Prevalent subspecies is MAA where high percentage of strains have inducible macrolide resistance. No other unknown genetic mutation was present in our isolates that can cause inducible macrolide resistance which is important for treatment patients with $\mathrm{CF}$, where clarithromycin is first drug of choice.

\subsection{4}

doi: 10.15789/2220-7619-2018-4-6.54

\section{MOLECULAR FEATURES OF MYCOBACTERIUM TUBERCULOSIS ISOLATES FROM PATIENTS LIVING IN CLOSED CITY IN THE URAL REGION, RUSSIA}

T.V. Umpeleva ${ }^{1}$, N.I. Eremeeva ${ }^{1}$, K.V. Belousova ${ }^{1}$, D.V. Vakhrusheva ${ }^{1}$, A.A. Vyazovaya ${ }^{2}$

${ }^{I}$ National Medical Research Center of Tuberculosis and Infectious Diseases, Ekaterinburg, Russia; ${ }^{2}$ St. Petersburg Pasteur Institute, St. Petersburg, Russia

Novouralsk is a closed town in the Sverdlovsk region, Middle Ural area in Russia, with a total population of 81500 and travel and residency restrictions. We aimed to identify the molecular-epidemiological features of $M$. tuberculosis circulating in Novouralsk under these specific conditions.

A total of $87 \mathrm{M}$. tuberculosis clinical isolates obtained between 2013 and 2016 from TB patients living in Novouralsk town were analyzed. According to clinical data, $34(39.1 \%)$ of TB patients were HIV-infected. 53 $(60.9 \%)$ of TB cases were newly diagnosed. Using realtime PCR we divided M. tuberculosis clinical isolates into Beijing/non-Beijing genetic groups. Beijing genotype variant B0/W148 was detected by multiplex PCR assay. VNTR loci MIRU26 and QUB26 were used for subtyping Beijing strains. Spoligotyping was used for further subtyping non-Beijing isolates. Drug susceptibility testing for first and second line drugs was performed by absolute consentration method.

Genotyping identified the predominance of the Beijing genotype isolates $(75.5 \%)$, among new TB cases, that is almost $20 \%$ higher than the average for the Ural region $(\mathrm{p}<0.05) .52 .8 \%$ isolates belonged to variant Beijing B0/W148. The majority of Beijing isolates $35(40.2 \%)$ had seven copies in MIRU26 and QUB-26 loci. Nine (10.3\%) Beijing B0/W148 isolates had 2 copies in QUB26 locus that was unusual for this genetic cluster; six patients from this group had TB/HIV co-infection. Seven $(8.0 \%)$ of non-Beijing isolates belonged to SIT35 spoligotype (Ural family). $20.7 \%$ of patient had prison history and $72.2 \%$ of them were infected with B0/W148 genotype. The MDR prevalence rate was higher than in Sverdlovsk region $(66 \%$ vs $43.9 \%, \mathrm{p}<0.05)$ and MDR status was associated with the Beijing B0/W148 genotype (94\% and $6 \%$ of its isolates were MDR and polyresistant, respectively).

Epidemiological situation with TB in Novouralsk is characterized by high level of TB/HIV co-infection, predominance of Beijing B0/W148 isolates, which is an underlying reason of high level of MDR-TB.
PREVALENCE AND DIVERSITY OF NONTUBERCULOUS MYCOBACTERIA IN DIFFERENT REGIONS OF THE RUSSIAN FEDERATION

V. Ustinova ${ }^{1,2}$, T. Smirnova ${ }^{1}$, E. Larionova ${ }^{1}$, I. Andrievskaya ${ }^{1}$, E. Kiseleva ${ }^{1}$, S. Andreevskaya ${ }^{1}$, L. Chernousova

${ }^{1}$ Central Tuberculosis Research Institute, Moscow, Russia; ${ }^{2}$ LLC Syntol, Moscow, Russia

Today, more attention is being paid worldwide to the nontuberculous mycobacteria (NTM) infections due to their increase in various regions of the world. The prevalence of different NTM species depends on the geographical location. The prevalence and distribution of the NTM species in Russian Federation have not been sufficiently studied to date.

The objective of this study was to demonstrate the diversity of NTM species isolated from patients in different regions of the Russian Federation. NTM were isolated from solid and liquid media from patients with suspected tuberculosis/ mycobacteriosis in the period from July, 2013 to June, 2017 and identified using GenoType Mycobacterium CM/AS assay (Hain Lifescience, Germany) and real-time PCR assay co-developed with Syntol LLC (Moscow, Russia). Seventeen NTM species were identified in 1400 cultures from 876 patients. Exact species was identified for isolates from 840/876 (95.89\%) patients. The prevalence of slowly growing NTM was $76.5 \%$ (643/840). The most common species was $M$. avium $(223 / 846,25.5 \%)$. Also the high incidence rate (in descending order) has been shown for M.gordonae (115/846, 13.12\%), M. lentiflavum (113/846, 12.9\%), M. intracellulare $(80,9.13 \%)$, M. fortuitum (83/846, 9.47\%), M. kansasii $(62 / 846,7.1 \%)$, M. abscessus (52/846, 5.9\%) and M. xenopi (31/846, 3.53\%). 16 of 17 identified species were detected in Moscow region. This may be due to a large number of cases analyzed in this region in comparison with other regions. It was shown that NTM species distribution in Central Federal District, European part of Privolzhsky Federal District and Kaliningrad (Northwest Russia) was similar to European countries: MAC 33-39\%, M. gordonae - 10-20\% and M. fortuitum - 5-13\%. The species distribution of NTM for neighboring Syktyvkar and Perm (North of European Russia) was similar and was characterized by high rates of $M$. fortuitum infection and low rates of $M$. avium infection. NTM, isolated from the border city of Khanty-Mansiysk were also characterized by low occurrence of $M$. avium and prevalence of $M$. gordonae.

To conclude, during the study period, 1400 samples collected from 876 patients in 6 federal districts were analyzed. The greatest species diversity - 16 NTM species - was shown for Moscow region. In most of the regions anayzed, slow-growing NTMs prevailed and the most abundant were species belonging to MAC complex. However, even on a relatively small number of observations, some regional features can be observed.

\subsection{6 doi: 10.15789/2220-7619-2018-4-6.56 MOLECULAR CHARACTERIZATION OF MYCOBACTERIUM BOVIS ISOLATES FROM CATTLE IN BULGARIA}

V. Valcheva ${ }^{1}$, T. Savova-Lalkovska ${ }^{2}$, A. Dimitrova ${ }^{2}$, H. Najdenski ${ }^{1}$, M. Bonovska ${ }^{1}$

${ }^{\prime}$ The Stephan Angeloff Institute of Microbiology, BAS, Sofia, Bulgaria; ${ }^{2}$ National Diagnostic and Research Veterinary Medical Institute, Sofia, Bulgaria

The observation of the population dynamics, diversity of genotypes and dissemination of Mycobacterium bovis strains in Bulgaria and neighboring countries play 
an important role in the modern epidemiological investigations of tuberculosis in animals at the regional and international level. Bovine tuberculosis represents a significant economic burden to the agriculture of the affected countries. From 2000 to 2015 the disease shows cyclicity in private farms in different regions of Bulgaria. This study is a first molecular investigation of animal tuberculosis in the veterinary medicine in country. The macroscopic and microscopic observation of 35 diagnostic materials from slaughtered cattle, received in the National Reference Laboratory of animal tuberculosis were studied with the three molecular methods: RD4PCR, spoligotyping and MIRU-VNTR. In 27 of the examined lymph nodes we found specific lesions for bovine tuberculosis. The findings were confirmed bacteriologically and by conventional PCR. To differentiate $M$. bovis from other $M$. tuberculosis complex subtypes, we used primers flanking specific deletion (RD4) in the genome of $M$. bovis and obtained the $446 \mathrm{bp}$ DNA product. The spoligotyping subdivided the strains into 3 spoligotypes shared by two to 20 strains. Further molecular investigations of $M$. bovis strains are needed to characterize the genetic diversity and population structure of $M$. bovis strains isolated from cattle in Bulgaria. New information will be added to the global database in the field of molecular epidemiology of the prevalence of $M$. bovis strains in the cattle population in Bulgaria, which will allow comparative analysis with data from the Balkan region and Europe.

Acknowledgements: This work was supported by the grant DN16/12 of the National Science Fund, Bulgaria.

\section{INTERNATIONAL VALIDATION OF ANALYSIS PIPELINES FOR WHOLE GENOME SEQUENCING DATA OF MYCOBACTERIUM TUBERCULOSIS ISOLATES}

D. van Soolingen ${ }^{1}$, R. Jajou ${ }^{1}$, T. Kohl ${ }^{2}$, T. Walker ${ }^{3}$, S. Niemann ${ }^{2}$, H. de Neeling ${ }^{1}$, R. Anthony ${ }^{1}$

${ }^{1}$ National Institute for Public Health and the Environment (RIVM), Bilthoven, The Netherlands; ${ }^{2}$ German Center for Infection Research, Borstel Site, Borstel, Germany; ${ }^{3}$ Nuffield Department of Medicine, University of Oxford, John Radcliffe Hospital, Oxford, United Kingdom

The aim of this multicenter study was to validate and compare different pipelines used for analysis of the Whole Genome Sequencing (WGS) data of Mycobacterium tuberculosis isolates.

All 535 M. tuberculosis isolates of culture positive cases in the Netherlands in 2016, were subjected to WGS, in addition to the routine application of VNTR typing. Transmission suggested on basis of identical VNTR profiles of cases in 2016 was further investigated by municipal health services and 41 epi-links were traced. Fastq.gz files of all 535 samples were analysed in four different WGS pipelines to facilitate international comparison: 1) SNP-based method at the RIVM/Bilthoven/ The Netherlands; 2) SNP-based method at Oxford University/UK; 3) SNP-based method and 4) cgMLST at Borstel/Germany.

In all pipelines, shorter than $12 \mathrm{SNP}$ distances between the 41 epi-linked cases was observed. One epi-linked pair revealed a higher genetic distance of 27 SNPs in the Bilthoven pipeline, due to poor sequence quality resulting in low coverage. In general, the genetic distances between isolates of the epi-linked cases were smaller in the Oxford and Borstel pipelines ( $0-3$ SNPs), than in the Bilthoven pipeline (1-11 SNPs). All pipelines clustered roughly the same cases, more isolates without identified epi-links were clustered in the Oxford $(n=34)$ and both Borstel pipelines $(n=32$ in the SNP pipeline and $n=39$ in the cgMLST) than the Bilthoven pipeline $(n=29)$.

Also, some cases not clustered by VNTR were clustered by WGS. Patient characteristics revealed that in some of these pairs of cases an epi-link, missed by VNTR typing, was likely.

Several differences were observed among the pipelines with regard to the version of reference genome used, software used for mapping and SNP calling, (repetitive) regions excluded in the analysis, the minimum number of reads to support SNPs, and the minimum allele frequencies. The RIVM pipeline was adapted in the light of these results to function more in line with other international laboratories pipelines, facilitating the comparability of results.

International standardization on all these variables is necessary, and subsequently on the SNP cut-off to be applied to WGS clustering, to allow international-laboratory comparison of WGS data and reliable investigation of cross-border transmission.

6.58

doi: 10.15789/2220-7619-2018-4-6.58

\section{RNA-BASED DRUG SUSCEPTIBILITY TESTING OF MYCOBACTERIUM TUBERCULOSIS}

A. Van den Bossche ${ }^{1}$, R. Bhattacharyya ${ }^{2}$, J.Y. Coppee ${ }^{3}$,

L. Rigouts ${ }^{4}$, A. Baulard ${ }^{5}$, A. Vodolazkaia ${ }^{6}$, D. Hung ${ }^{2}$,

V. Mathys ${ }^{1}$, P.J. Ceyssens ${ }^{1}$

${ }^{1}$ Division of Bacterial Diseases, Sciensano, Brussels, Belgium; ${ }^{2}$ Harvard Medical School, Boston, United States; ${ }^{3}$ Institut Pasteur, Paris, France; ${ }^{4}$ Institute of Tropical Medicine, Antwerp, Belgium; ${ }^{5}$ Center for Infection and Immunity of Lille, Institut Pasteur de Lille, France; ${ }^{6}$ Laboratory of Medical Microbiology, Sciensano, Brussels, Belgium

Multidrug resistant tuberculosis (MDR-TB) is one of the major WHO health concerns. One of the challenges that hampers the effective response to MDR-TB is the long turnaround time of phenotypic Drug Susceptibility Testing (DST) of Mycobacterium tuberculosis. To counter this, new fast and sensitive DNA-based methods were successfully introduced over the last years. However, these (a) are based on the knowledge on resistance mutations, (b) do not distinguish living from dead cells, (c) ignore all intrinsic resistance mechanisms, and (d) ignore the influence of compensatory mutations.

We introduce a next-generation diagnostic test based on quantification of drug-specific RNA biomarkers. The basic principle is that a brief antibiotic exposure triggers specific transcriptional responses in susceptible, but not in resistant, microbes within a few hours. This has the advantage that long culture-dependent steps are avoided, yet the resistance phenotype is detected independent of the specific cause of resistance.

First, the global transcriptional response of two M. tuberculosis strains to 10 anti-TB drugs was determined using RNAtaq-Seq. A set of highly responsive genes was selected for each drug and RNA-targeting probes were designed.

Next, the RNA-based DST was developed in 96 well format. In short, $200 \mu \mathrm{l}$ of a positively flagged MGIT ${ }^{\mathrm{TM}}$ (BD) culture is spiked with a drug, while a replicate is incubated in absence of the drug. Multiplex mRNA quantification is performed directly on crude cell lysates using a combination of the bead-based $\mathrm{MagPix}^{\mathrm{TM}}$ (Luminex) and Quantigene ${ }^{\mathrm{TM}}$ Plex (Thermo Fisher) technology. 